\title{
Evaluation of Basophil CD203c as a Predictor of Carboplatin-Related Hypersensitivity Reaction in Patients with Gynecologic Cancer
}

\author{
Takuya Iwamoto, ${ }^{* a}$ Atsushi Yuta, ${ }^{b}$ Tsutomu Tabata ${ }^{c}$ Hiroko Sugimoto, ${ }^{a}$ Esteban Cesar Gabazza, ${ }^{d}$ \\ Hiroyuki Hirai, ${ }^{e}$ Shigeyuki Kojima, ${ }^{e}$ and Masahiro Okuda ${ }^{a}$ \\ ${ }^{a}$ Department of Pharmacy, Mie University Hospital; ${ }^{b}$ Department of Otorhinolaryngology-Head and Neck Surgery, \\ Mie University, Graduate School of Medicine; ${ }^{c}$ Department of Obstetrics and Gynecology, Mie University, Graduate \\ School of Medicine; ' Department of Immunology, Mie University, Graduate School of Medicine; Tsu, Mie 514-8507, \\ Japan: and ${ }^{e} R \& D$ Center, BML, Inc.; Kawagoe, Saitama 350-1101, Japan.
}

Received February 13, 2012; accepted June 4, 2012

The incidence of hypersensitivity reaction (HR) to carboplatin has been reported to increase after repeated use of the drug. However, a reliable ex vivo test to predict HR to carboplatin is not currently available. We evaluated the clinical usefulness of measuring basophil CD203c to predict carboplatin-related HR in this prospective case-control study conducted at Mie University Hospital between October 2009 and September 2010. Eleven patients had history of carboplatin-related HR within the past 3 years, and 19 had no history of HR after receiving more than 5 courses of carboplatin therapy. Six of these 19 patients developed carboplatin-related HR during the study period. The CD203c+ basophils (\%) and the mean fluorescence intensity (MFI) were analyzed on a flow cytometer and compared between patients with and without HR. Changes in the CD203c expression on basophils before and after HR were also assessed in patients who developed HR during the study period. The median CD203c+ basophils $(\%)$ and $\triangle M F I$ after 30 -min exposure to $50 \mu \mathrm{g} / \mathrm{mL}$ carboplatin were significantly higher in patients with HR $(3.5 \%$ and $\Delta$ MFI 9.0) compared with those without $(2.2 \%$ and $\triangle$ MFI 0.4$)(p<0.05)$. In particular, these values were significantly higher in patients with grade 4 anaphylaxis $(10.6 \%$ and $\triangle$ MFI 22.0$)$. All five patients who developed grade $2-4$ anaphylaxis during the study period had high CD203c+ basophils (\%) and/or increased $\triangle$ MFI on the day before HR. The results suggest that basophil CD203c may be a promising biomarker for the prediction of severe carboplatinrelated anaphylaxis.

Key words basophil activation test; CD203c; hypersensitivity reaction; carboplatin

Carboplatin is one of the most commonly used antineoplastic drugs for gynecologic cancers. One complication of carboplatin is hypersensitivity reaction (HR), ${ }^{1-3)}$ and more severe side effects such as hypertension, hypotension, vomiting, dyspnea and wheezing, are the cause of discontinuation of the drug. The reported incidence of HR in patients who received more than 7 courses of carboplatin is $26.7 \%,{ }^{1)}$ which is similar to that reported previously by our group (23.2\%) in patients with gynecologic cancer. ${ }^{4}$ Although the risk of carboplatinrelated HR is thought to be associated with previous history of drug allergy, prolonged platinum-free interval or high dose of carboplatin, ${ }^{2-5)}$ these parameters are not very sensitive for the prediction of HR. Intradermal injection of carboplatin has also been used for the diagnosis of carboplatin-related HR, and the reported sensitivity and false-positivity of the test are $85.7 \%$ and $1.5 \%$, respectively, in patients with gynecologic cancer. ${ }^{6}$ Another study reported that 5 out of 7 patients had carboplatin-related HR after positive intradermal tests. ${ }^{7)}$ The intradermal carboplatin test may be useful, however, it is not preferable because of the risk of skin damage, environmental contamination and exposure of the medical and nursing professionals to anticancer drugs.

Basophils play important roles in allergic diseases through the release of inflammatory mediators such as histamine and by the production of $\mathrm{T}$ helper 2 (Th2) cytokines such as interleukin-4 (IL-4) and IL-13. ${ }^{8}$ Upon stimulation with specific allergens that cross-link immunoglobulin E (IgE) bound to its high-affinity receptor, basophils rapidly express surface

The authors declare no conflict of interest. molecules such as CD203c and CD63. Flow cytometry-based tests for peripheral blood basophils can easily quantify these in vitro reactions, which presumably represent their in vivo activity for hymenoptera, latex, food and drug allergies. ${ }^{9-13)}$ The sensitivity of the basophil activation test for the diagnosis of drug allergy has been reported; the reported sensitivities for the diagnosis of allergy to $\beta$-lactams, ${ }^{14-16)}$ quinolones $^{17)}$ and rocuronium ${ }^{18)}$ are $33-67 \%, 71.1 \%$ and $80 \%$, respectively. In addition, increased CD63 expression on basophils has been reported in a patient with cisplatin allergy. ${ }^{19)}$ Cisplatin is a platinum analogue that resembles carboplatin in its pharmacological profile. Some studies suggested that CD203c might be a more specific marker of activated basophils than CD63, ${ }^{14,20}$ however, to date, it is not clear whether overexpression of basophil CD203c exists in patients with carboplatin-related HR.

In this study, we hypothesized that quantification of basophil CD203c may be useful for the prediction of carboplatinrelated HR. To test the hypothesis, we measured basophil CD203c expression and evaluated the sensitivity and specificity of the test in patients with gynecologic cancer.

\section{MATERIALS AND METHODS}

Patients This prospective study comprised patients who were admitted to Mie University Hospital between October 2009 and September 2010. The entry criteria were as follows: diagnosis of gynecologic cancer, past history of carboplatin therapy for more than 5 courses or history of carboplatin-related HR within the past 3 years. At each hospital visit, blood samples were obtained from the patients on the day before 


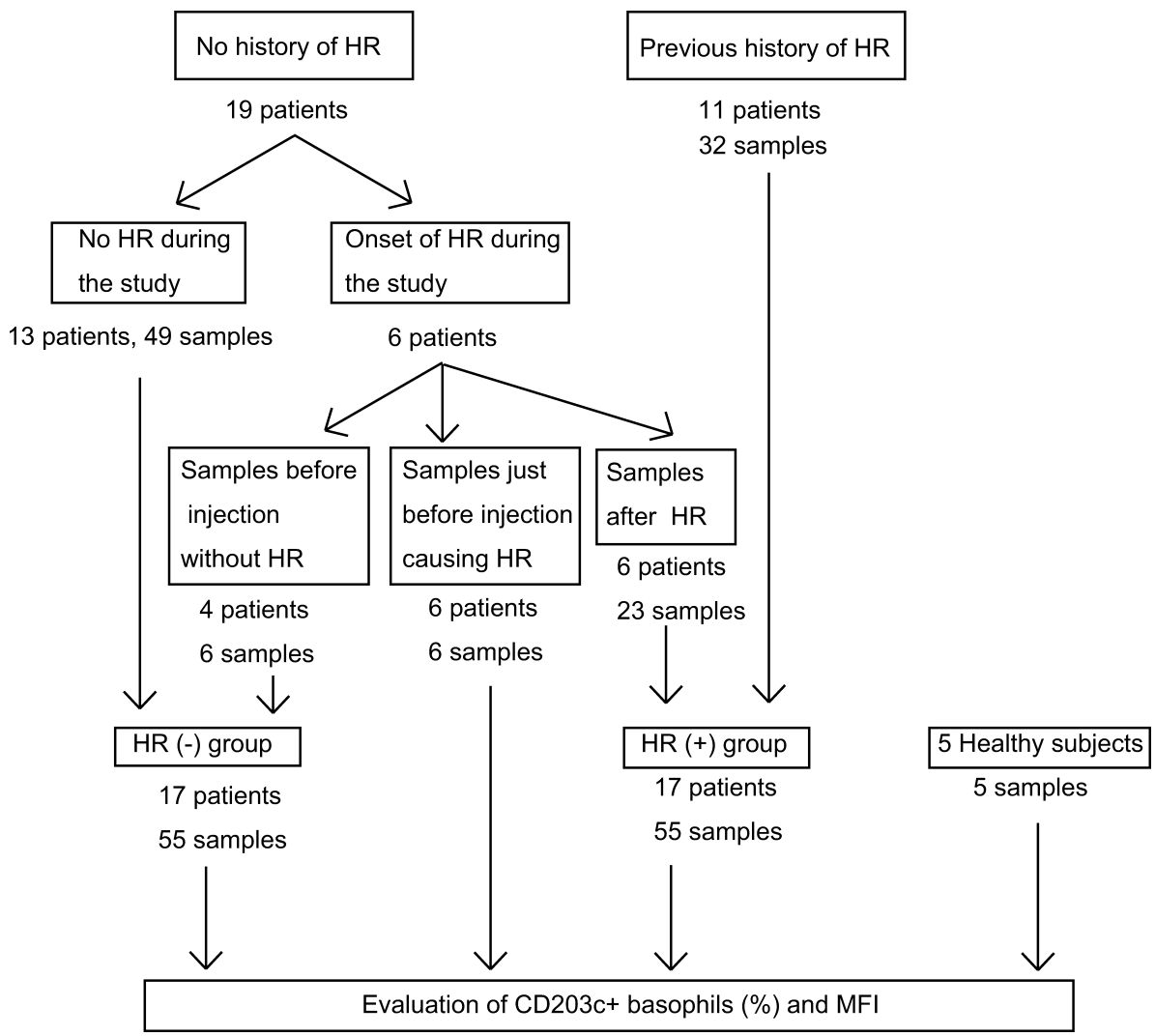

Fig. 1. Schematic Flow Diagram of Recruitment of $\mathrm{HR}(+)$ and $\mathrm{HR}(-)$ Patients for the Basophil Activation Test HR: hypersensitivity reaction, MFI: mean fluorescence intensity.

carboplatin administration. Premedication treatments, histamine $\mathrm{H}_{1}$-receptor blocker and/or corticosteroid, were started after blood sampling in all patients treated with carboplatin. Five healthy subjects who have not received carboplatin were enrolled in this study to confirm the homogeneity of basophil activation between $\mathrm{HR}(-)$ patients and healthy subjects. This study protocol was reviewed and approved by the Ethics Committee of Mie University, and a written informed consent was obtained from each subject.

Measurement of CD203c Expression on Basophils The basophil activation test was carried out within $24 \mathrm{~h}$ of blood sampling. A commercial kit (Allergenicity Kit, Beckman Coulter, Fullerton, CA, U.S.A.) was used for quantification of basophil CD203c expression according to the instructions supplied by the manufacturer. ${ }^{21)}$ Briefly, whole blood anticoagulated with ethylenediaminetetraacetic acid (EDTA) was incubated with various concentrations $(500,50,5 \mu \mathrm{g} / \mathrm{mL})$ of carboplatin (Paraplatin, Bristol-Myers Squibb Company, Tokyo), Anti-IgE antibody $(4 \mu \mathrm{g} / \mathrm{mL})$ as positive control, or $5 \%$ dextrose solution (a vehicle) as negative control for $30 \mathrm{~min}$ or $3 \mathrm{~h}$ after the addition of an appropriate amount of calcium solution to override the chelating effect of EDTA. R-Phycoerythrin-Cyanine 7 (PC7)-conjugated anti-CD3, Fluorescein isothiocyanate (FITC)-conjugated anti-CRTH2 and R-Phycoerythrin (PE)-conjugated anti-CD203c antibodies were added to the reaction mixture for last $30 \mathrm{~min}$. The red blood cells in the sample were lysed and then leukocytes in the sample were analyzed on a flow cytometer FACSCalibur (Becton Dickinson Biosciences, Sparks, MD, U.S.A.). Basophils were identified by characteristic forward and side scatter, by the expression of
CRTH2 and by the absence of CD3.

Evaluation of CD203c Expression on Basophils as a Predictor of Carboplatin-Related HR Positive CD203c basophils were identified by the higher fluorescence intensity (CD203c expression) compared with that of $2 \%$ of individual unstimulated cells (negative control). For each subject, we measured the mean fluorescence intensity (MFI) using total basophils, including both CD203c-positive and -negative basophils. $\triangle$ MFI was calculated as difference from MFI of negative control of each subject. We compared the percentage of CD203c-positive basophils and $\triangle$ MFI between $\mathrm{HR}(+)$ and $\mathrm{HR}(-)$ groups using the mean values for each subject. We also compared the expression of CD203c on basophils between groups classified on the basis of severity of anaphylaxis. ${ }^{22)} \mathrm{Pa}-$ tients with mild rash, pruritis, and/or flushing were classified as grade 1, those with vomiting, edema, and/or diffuse hives were classified as grade 2, those with chest pain were grade 3 , and patients who developed hypertension, hypotension, diarrhea, dyspnea, and/or wheezing were classified as grade 4 anaphylaxis.

To evaluate the validity of $\mathrm{CD} 203 \mathrm{c}$ as a predictor of anaphylaxis, changes in the CD203c expression on basophils before and after anaphylaxis were assessed in 6 patients who developed HR during the study period.

Statistical Analysis Nonparametric Wilcoxon's rank sum test was used to assess differences between patients with and without carboplatin-related HR. Nonparametric Dunnett's multiple comparison test was used to compare variables among the three groups. We also conducted the area under receiver-operator characteristics (ROC) curve analyses to 
Table 1. Characteristics of the Patients with No History of HR and Previous History of HR at the Entry of the Study

\begin{tabular}{|c|c|c|}
\hline & No history of HR & Previous history of HR \\
\hline No. of patients $(n)$ & 19 & 11 \\
\hline Age $(\text { years })^{a)}$ & $61(37-80)$ & $51(48-81)$ \\
\hline \multicolumn{3}{|l|}{ Organ with cancer $(n)$} \\
\hline Ovary & 17 & 10 \\
\hline Endometrium & 1 & 1 \\
\hline Uterus (uterine sarcoma) & 1 & 0 \\
\hline Doses of carboplatin ${ }^{a}$ & $450(300-760)$ & $600(375-800)$ \\
\hline Courses of carboplatin therapy & $5(10-42)$ & $10(5-20)$ \\
\hline
\end{tabular}

HR: hypersensitivity reaction. a) Data are expressed as median values (minimum-maximum).

estimate the accuracy and cutoff values for certain metric variables for the diagnosis of carboplatin-related HR. All statistical analyses were carried out using SAS software (version 9.1.3; SAS Institute Japan, Tokyo). The $p$ values were twosided and $p<0.05$ was considered statistically significant.

\section{RESULTS}

Patients Thirty female patients with gynecologic cancer were enrolled in this case-control study. Eleven patients had a history of carboplatin-related HR, and 19 had previously received intravenous injection of carboplatin but did not have any history of carboplatin-related HR. Age, organ with cancer, dose of carboplatin, and courses of carboplatin received at study entry are shown in Table 1. Among the 19 patients with negative history of HR, 6 showed carboplatin-related HR during the study period (Fig. 1). Five healthy subjects with no history of carboplatin administration served as controls.

Induction of CD203c Expression on Basophils by Carboplatin As shown in Fig. 1, CD203c expression on basophils was evaluated in 17 patients (55 samples) of the HR(+) group (including 11 with previous history of HR and 6 patients who

A developed HR during the study), 17 patients (55 samples) of the HR(-) group (including 13 with negative history of HR and 4 who developed HR during the study) and 5 healthy subjects (5 samples). Table 2 shows the expression of CD203c on basophils $30 \mathrm{~min}$ after carboplatin exposure. A cutoff value $(8 \%)$ of $4 \times$ negative control was applied after anti-IgE antibody exposure (positive control). Twelve out of 115 samples were excluded from the study because the positive control was $<8 \%$. The percentage of activated basophils was significantly higher in the $\mathrm{HR}(+)$ group (median 3.5\%) than in the HR(-) group (median $2.2 \%$ ) after exposure to $50 \mu \mathrm{g} / \mathrm{mL}$ carboplatin $(p=0.037)$. The $\triangle$ MFI of $\mathrm{CD} 203+$ basophils was also significantly higher in the $\mathrm{HR}(+)$ group than in the $\mathrm{HR}(-)$ group after exposure to $50 \mu \mathrm{g} / \mathrm{mL}$ carboplatin (median $\Delta \mathrm{MFI}$ : $9.0 \mathrm{vs}$. 0.4) $(p=0.047)$.

ROC curve analysis demonstrated that the cutoff value of CD203c+ basophils (\%) was 3.5\% after exposure to $50 \mu \mathrm{g} / \mathrm{mL}$ carboplatin for $30 \mathrm{~min}$, and the calculated area under the ROC curve $(A U C)$ was 0.73 . Using this cutoff value, the sensitivity, specificity and accuracy of the CD203c+ basophil (\%) test to detect carboplatin-related HR were $56.3 \%, 86.7 \%$ and $70.1 \%$, respectively. In the same way, the cutoff value of $\Delta \mathrm{MFI}$ was

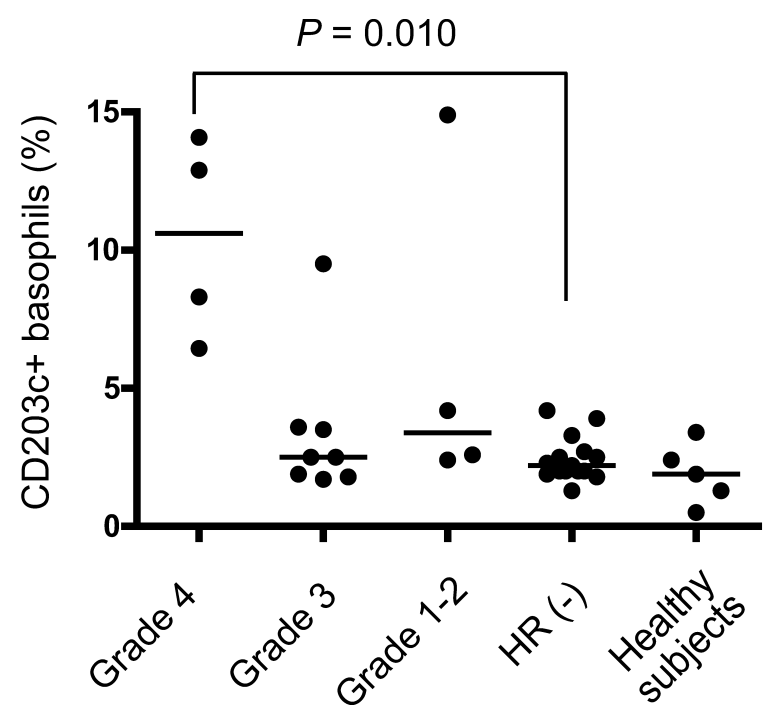

\section{B}

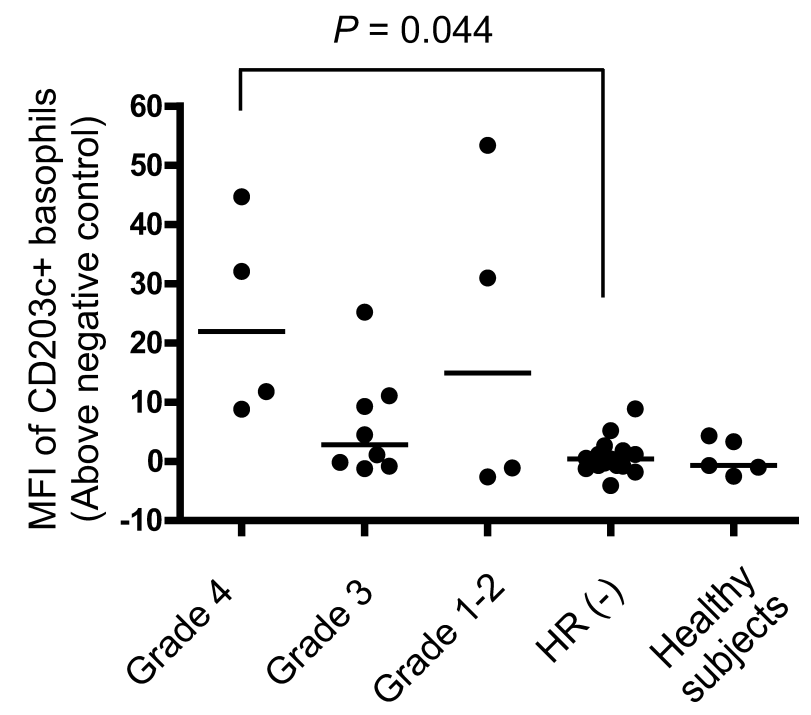

Fig. 2. Percentage of CD203c-Positive Basophils (A) and $\Delta$ MFI of CD203c on Basophils (B) after 30 min Exposure to $50 \mu \mathrm{g} / \mathrm{mL}$ Carboplatin

The severity of anaphylaxis was evaluated using the scoring system of Sampson. ${ }^{22}$ The numbers of patients were as follows: anaphylaxis grade $4(n=4)$, grade 3 ( $\left.n=8\right)$, grade 1-2 $(n=4), \operatorname{HR}(-): n=15$, and healthy subjects: $n=5$. The horizontal line represents the median value for the entire group. Statistical differences among groups were evaluated by Dunnett's multiple comparison tests. 

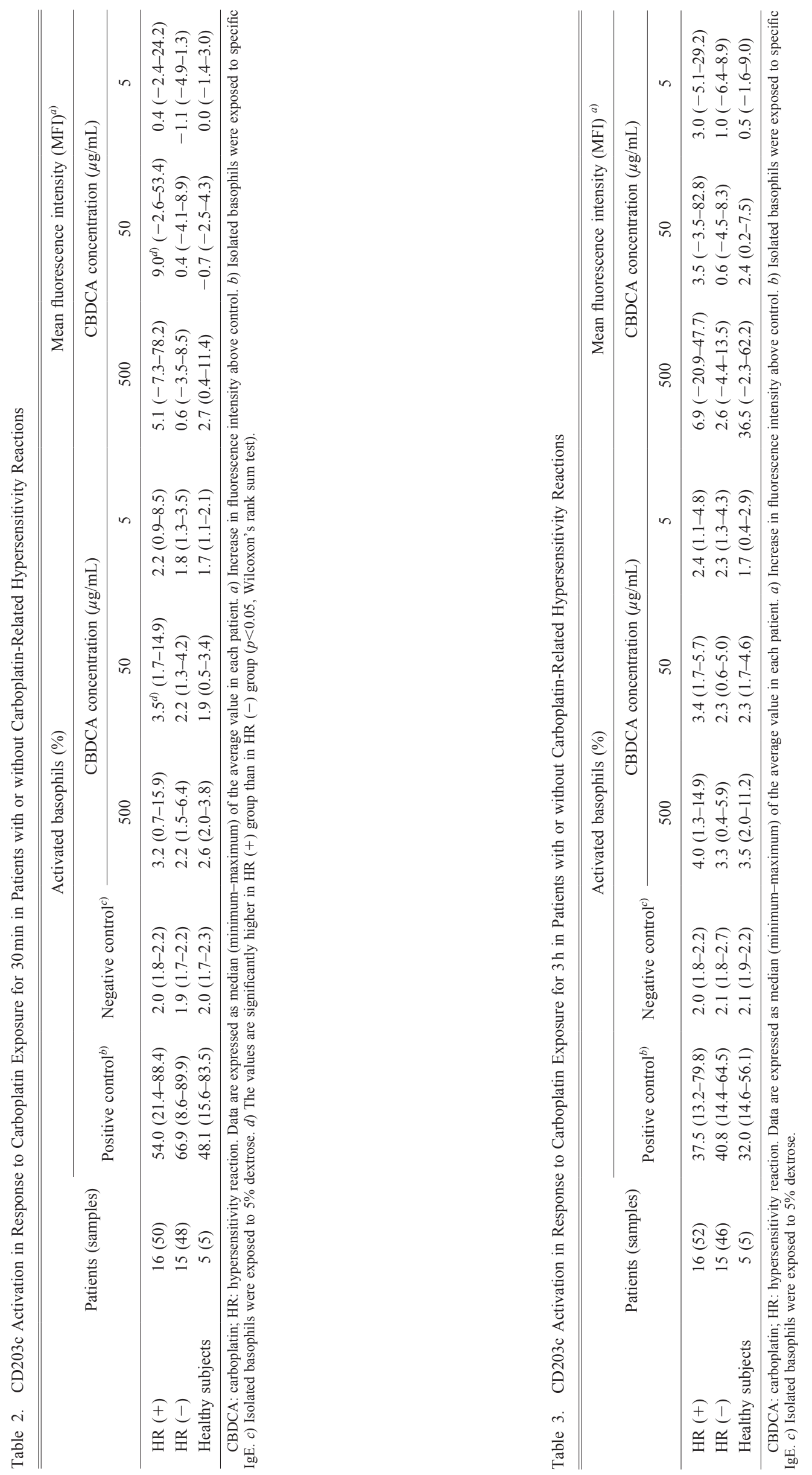
A

Patient 1 : grade 4 anaphylaxis

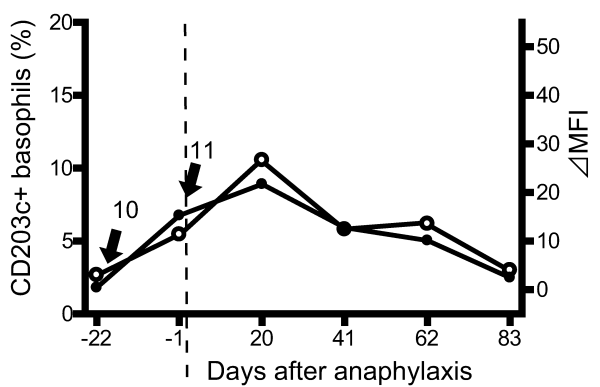

Anaphylaxis

C

Patient 3 : grade 3 anaphylaxis

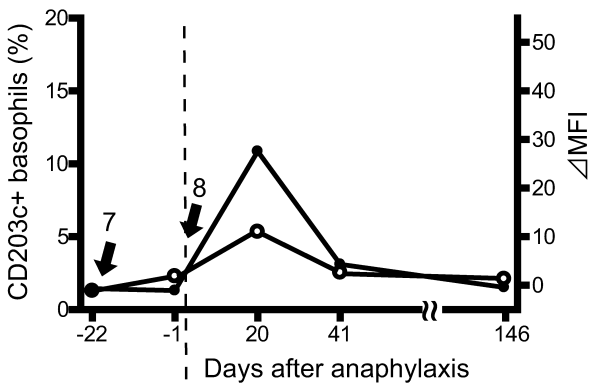

Anaphylaxis

E

Patient 5 : grade 2 anaphylaxis

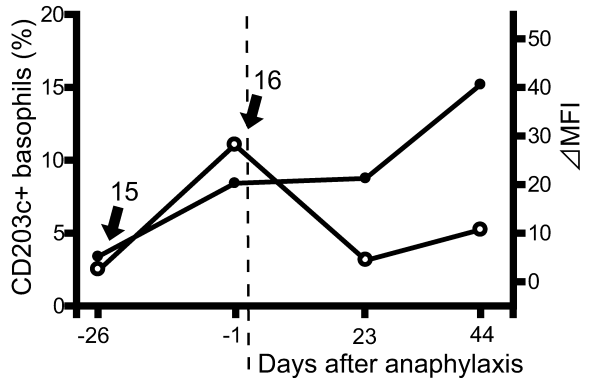

Anaphylaxis
B

Patient 2 : grade 4 anaphylaxis

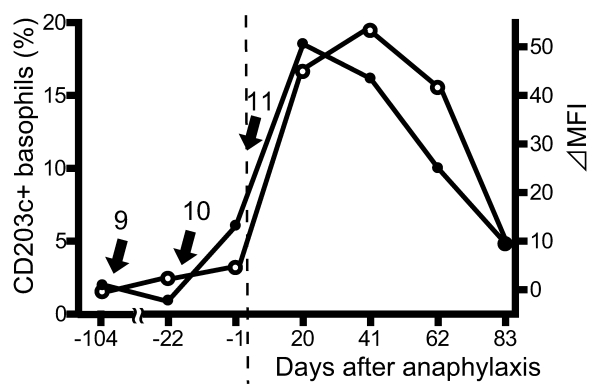

Anaphylaxis

D

Patient 4 : grade 3 anaphylaxis

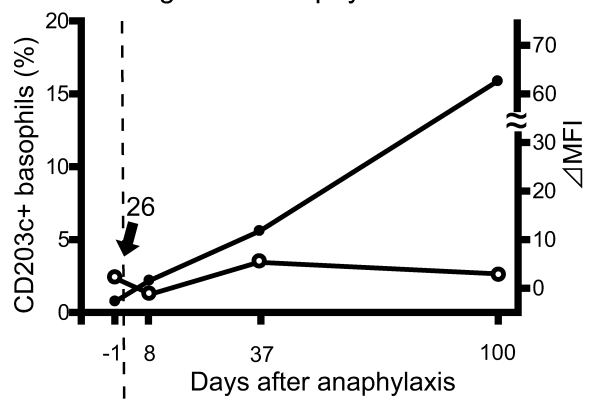

Anaphylaxis

Patient 6 : grade 1 anaphylaxis

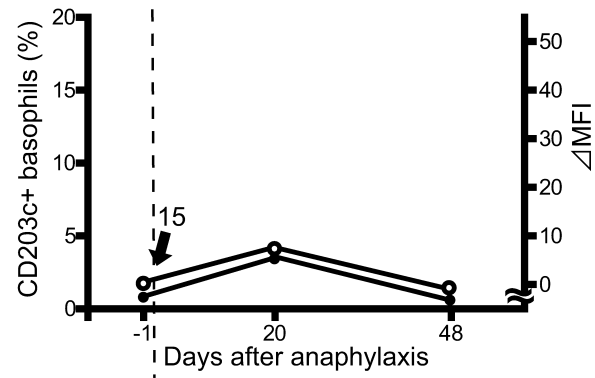

Anaphylaxis

Fig. 3. CD203c-Positive Basophils (\%) and $\triangle \mathrm{MFI}$ of CD203c before and after Anaphylaxis in 6 Patients with Carboplatin-Related Anaphylaxis

Blood samples were treated with carboplatin $(50 \mu \mathrm{g} / \mathrm{mL})$ for $30 \mathrm{~min}$ and then the expression of CD203c on basophils was evaluated. Open circles: percentage of activated basophils, solid circles: $\triangle$ MFI of CD203c on basophils. Numbers on arrows refer to the cycles of carboplatin administration.

7.0, and using this cutoff value, the sensitivity, specificity and accuracy were $56.3 \%, 93.3 \%$ and $74.2 \%$, respectively. The percentages of CD203c+ basophils (\%) in the positive and negative controls were not significantly different between the $\mathrm{HR}(+)$ and HR(-) groups (Table 2).

Table 3 shows the expression of CD203c on basophils $3 \mathrm{~h}$ after carboplatin exposure. Twelve out of 110 samples were excluded from the study because the percentage of activated basophils was less than $8 \%$ after anti-IgE antibody exposure. However, there was no significant difference in basophil activation between the $\mathrm{HR}(+)$ and $\mathrm{HR}(-)$ groups. ROC curve analysis demonstrated that the cutoff values of CD203c+ basophils $(\%)$ and $\triangle \mathrm{MFI}$ after exposure to $50 \mu \mathrm{g} / \mathrm{mL}$ carboplatin for $3 \mathrm{~h}$ were $3.4 \%$ and 6.0 , respectively. The calculated $A U C$ for CD203c+ basophils (\%) and $\triangle$ MFI were 0.69 and 0.65 , respectively.

Expression of CD203c on Basophils According to the Severity of Anaphylaxis The expression of CD203c on basophils was compared among patients with different grades of anaphylaxis. Although we did not perform skin tests to confirm hypersensitivity to carboplatin, all signs and symptoms of HR developed in our hospital during carboplatin infusion, and all patients with grade $\geq 2$ anaphylaxis received intravenous hydrocortisone immediately after anaphylactic reactions. The percentage of $\mathrm{CD} 203 \mathrm{c}+$ basophils at $30 \mathrm{~min}$ after exposure to $50 \mu \mathrm{g} / \mathrm{mL}$ carboplatin and its $\Delta$ MFI were significantly higher in patients with grade 4 anaphylaxis (median $10.6 \%$ and $\triangle$ MFI 22.0) compared with those of patients without HR 


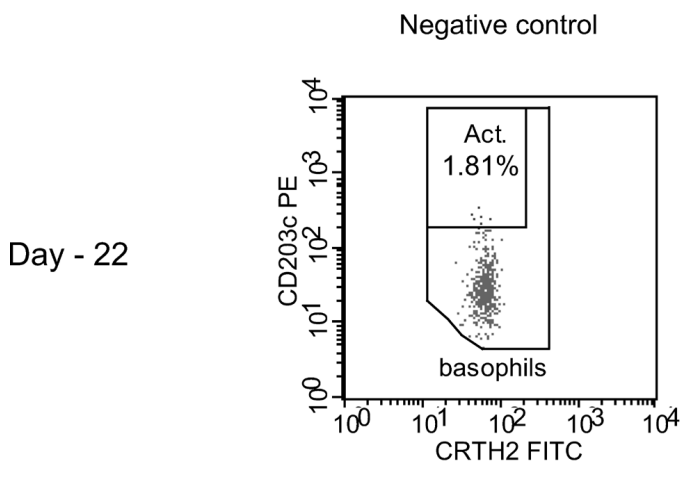

MFI of CD203c
Treated with Carboplatin for $30 \mathrm{~min}$

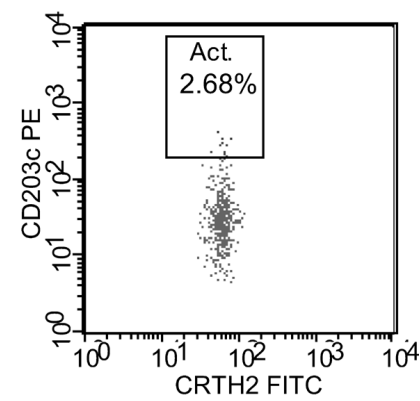

31.05
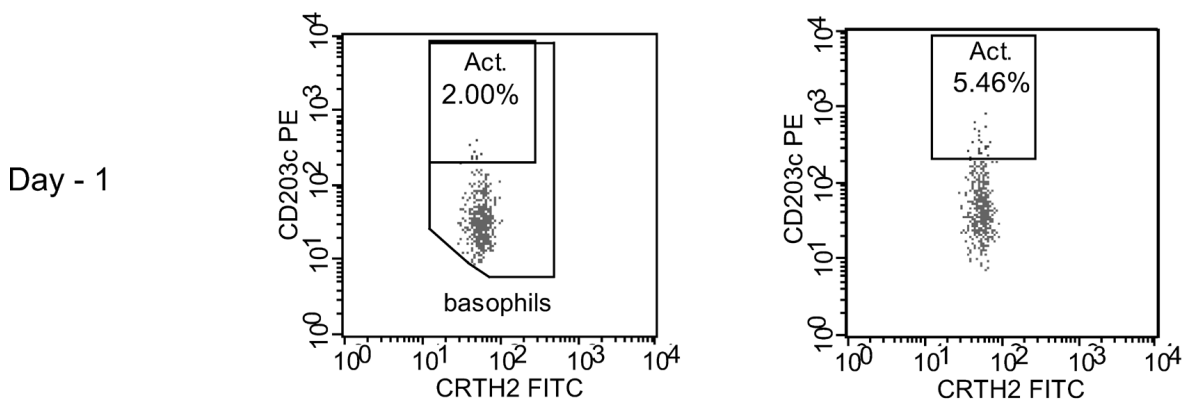

MFI of CD203c

35.15

$\triangle \mathrm{MFI} 15.28$

50.43

Fig. 4. Dot Plot Graphs of Basophil Activation by Carboplatin in Day-22 and Day-1 of Patient 1

Act. means activated basophils. In the negative control, whole blood anticoagulated with EDTA was incubated with $5 \%$ dextrose solution. Data were analyzed with CellQuest software (Becton Dickinson Biosciences). Basophils were showed by gating of the mononuclear cells, CD3 ${ }^{-}$and $\mathrm{CRTH} 2^{+}$.

(median $2.2 \%$ and $\triangle$ MFI 0.4$)(p=0.010$ and $p=0.044$, respectively) (Figs. 2A,B). In all patients with history of grade 4 anaphylaxis, the increases in $\mathrm{CD} 203 \mathrm{c}+$ basophils (\%) and $\triangle \mathrm{MFI}$ were more than $3.5 \%$ and 7.0 , respectively, which were the cutoff values calculated by ROC analysis.

Evaluation of CD203c Test as a Predictor of Carboplatin-Related HR The percentage of CD203c+ basophils and $\Delta$ MFI after exposure to $50 \mu \mathrm{g} / \mathrm{mL}$ carboplatin for $30 \mathrm{~min}$ were evaluated before and after anaphylaxis in six patients who developed HR during the study period (Fig. 3). Dot plot graphs analyzed on a flow cytometer in the patient 1 (day-22 and day-1) were shown in Fig. 4. The percentages of CD203c+ basophils and/or $\triangle$ MFI markedly increased at the onset of anaphylaxis in 5 patients with grade 2-4 anaphylaxis (Figs. $3 \mathrm{~A}-\mathrm{E}$ ), but they were not increased in one patient with grade 1 anaphylaxis (Patient 6) (Fig. 3F). $\triangle$ MFI on the day before anaphylaxis (day-1) increased in 3 patients (Patients 1, 2, 5, Figs. 3A, B, E), and the values were higher than the cutoff value ( $\triangle$ MFI 7.0). The CD203c+ basophils (\%) on the day before anaphylaxis were higher in Patient 1 with grade 4 anaphylaxis and Patient 5 with grade 2 anaphylaxis (Figs. 3A, E) than the cutoff value $(3.5 \%)$.

Longitudinal changes in the percentages of CD203c+ basophils and $\triangle \mathrm{MFI}$ after exposure to $50 \mu \mathrm{g} / \mathrm{mL}$ carboplatin for $3 \mathrm{~h}$ were also investigated in the same 6 patients (Fig. 5). The percentage of activated basophils and $\triangle \mathrm{MFI}$ on the day before anaphylaxis increased in four patients (Patients 1, 3-5), and these were higher than the cutoff values $(3.4 \%, \Delta \mathrm{MFI} 6.0) \mathrm{ob}$ served in the ROC analysis. The CD203c+ basophils (\%) and $\triangle \mathrm{MFI}$ in these patients ranged from 6.5 to $9.4 \%$ and from 7.0 to 26.8 , respectively.

\section{DISCUSSION}

Several studies have recently assessed the value of the basophil activation test using the expression of CD63 or CD203c for the diagnosis of drug allergy. ${ }^{13-18)}$ Some researchers suggested that CD203c is more sensitive than CD63 for the diagnosis of drug allergy. ${ }^{14,20)}$ This may be due to the fact that CD63 is not only a marker of activated basophils but also of platelets and leukocytes. In addition, adhesion of activated platelets to basophils may hamper the correct analysis of CD63 expression on basophils. ${ }^{23)}$ However, the CD203c-based basophil activation test is still limited compared with the CD63-based test, and the utility of CD203c-based test for the detection of platinum-induced allergy remains unclear.

We showed for the first time the usefulness of CD203cbased basophil activation test for the detection and prediction of severe carboplatin-related HR. The results showed that the most appropriate concentration of carboplatin for treating basophils is $50 \mu \mathrm{g} / \mathrm{mL}$ for $30 \mathrm{~min}$. When basophils were exposed to $500 \mu \mathrm{g} / \mathrm{mL}$ carboplatin for $30 \mathrm{~min}$, three samples of the HR(-) group showed positive results (activated basophils $6.4 \%, 5.2 \%, 4.3 \%$ ) and one of a healthy subject (MFI 
A

Patient 1 : grade 4 anaphylaxis

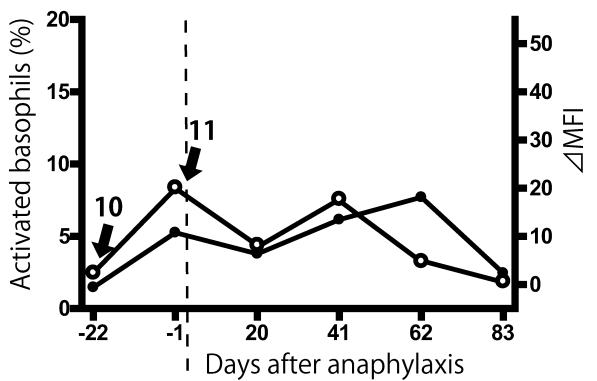

Anaphylaxis

C

Patient 3 : grade 3 anaphylaxis

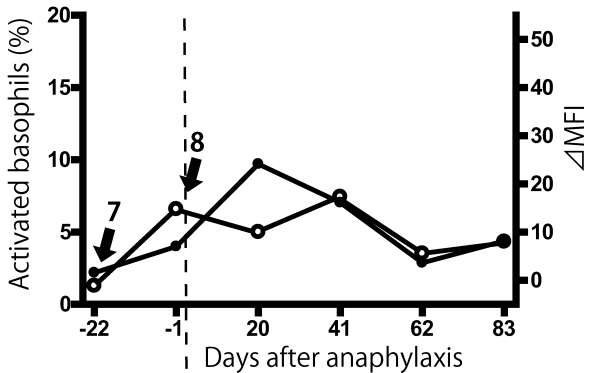

Anaphylaxis

E

Patient 5 : grade 2 anaphylaxis

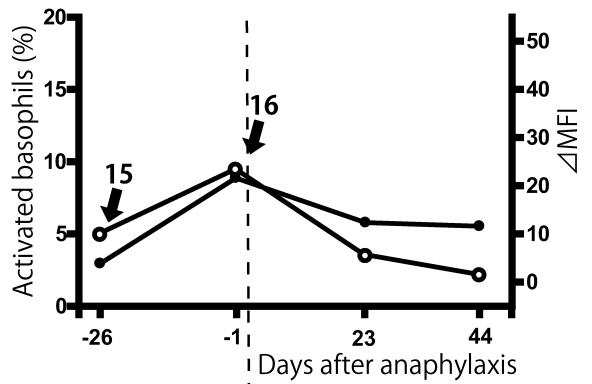

Anaphylaxis
B

Patient 2 : grade 4 anaphylaxis

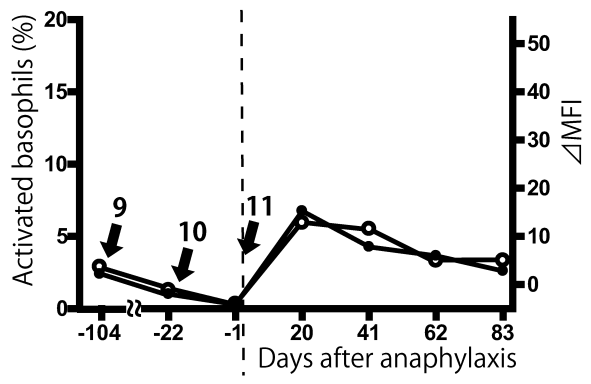

Anaphylaxis

D

Patient 4 : grade 3 anaphylaxis

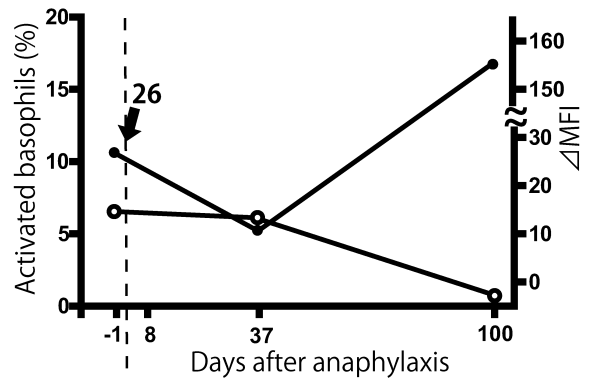

Anaphylaxis

F

Patient 6 : grade 1 anaphylaxis

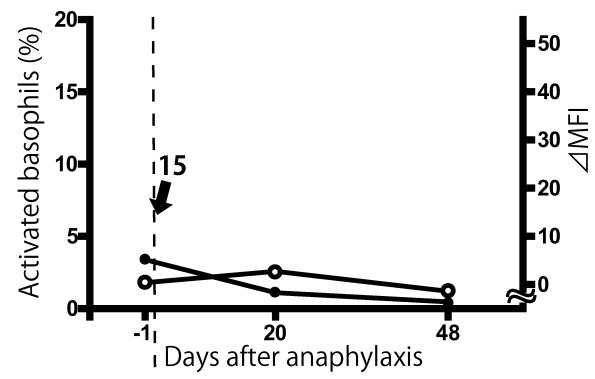

Anaphylaxis

Fig. 5. CD203c-Positive Basophils (\%) and $\Delta$ MFI of CD203c before and after Anaphylaxis in 6 Patients with Carboplatin-Related Anaphylaxis

Blood samples were treated with carboplatin $(50 \mu \mathrm{g} / \mathrm{mL})$ for $3 \mathrm{~h}$ and followed by evaluation of CD203c expression on basophils. Open circles: percentage of activated basophils, solid circles: $\triangle$ MFI of CD203c on basophils. Number on arrows refers to the cycles of carboplatin administration.

11.4). These false positive results lower the utility of the test for the prediction of carboplatin-related HR. The cytotoxicity of $500 \mu \mathrm{g} / \mathrm{mL}$ carboplatin appeared to have little effect on the test based on flow cytometric analysis using at least 500 intact cells and that anticancer drugs are known to cause DNA damage in immature cells during differentiation but not mature basophils. Exposure of blood basophils to $50 \mu \mathrm{g} / \mathrm{mL}$ carboplatin for $30 \mathrm{~min}$ induced a significant increase in CD203c+ basophils (\%) and $\triangle \mathrm{MFI}$ in patients with grade 4 anaphylaxis. All such patients had increased CD203+ basophils, and these levels were higher than the cutoff values calculated by ROC analyses. However, when all patients, irrespective of the grade of anaphylaxis, and non-responder (one patient) was included in the analysis, the sensitivities were $52.9 \%$ (9 out of 17 patients) both for $\%$ activated basophils and $\Delta \mathrm{MFI}$, which are relatively low compared with the previously reported sensitivity of intradermal test of $85.7 \%{ }^{6}$ )

The time interval between HR and CD203c analysis may influence the result of the basophil activation test. ${ }^{24)}$ This time interval in the 6 patients with false-negative test results was between $7 \mathrm{~d}$ and 25 months. One false-negative result was recorded at $7 \mathrm{~d}$ after grade 1 anaphylaxis. In the other 5 patients, CD203c+ basophils did not increase after both $30 \mathrm{~min}$ and $3 \mathrm{~h}$ exposure to carboplatin and the time interval between HR and CD203c analyses were longer than $48 \mathrm{~d}$. We also confirmed that fresh samples (obtained shortly after HR) from patients who developed grades 2-4 anaphylaxis during the study period are sensitive to the basophil activation test, whereas the 
positive basophils (\%) decreased below the cutoff value within 3 months in 4 patients. In the previous report regarding the basophil activation test for pyrazolones, which causes immediate IgE-mediated reactions, follow-up of the positive patients showed a decrease over time, with $60 \%$ becoming negative after 6 months. ${ }^{25)}$ Similar results were also reported in some positive cases of IgE mediated quinolone allergies. ${ }^{17)}$ Therefore, it is not clear whether basophil responsiveness is maintained over a long period even in an IgE-mediated reaction, and CD203c expression-based basophil activation test should be performed at the early stages of carboplatin-related HR for more precise results. Further longitudinal follow-up study is needed to confirm the effect of sampling timing on basophil activation test.

Although carboplatin is repeatedly used in patients with recurrent ovarian cancer until disease progression, the incidence of HR increases with repeated injections of carboplatin. Therefore, it is important to predict carboplatin-related HR for the safe management of cancer chemotherapy. Several studies have shown the usefulness of the basophil activation test, ${ }^{9-13)}$ but they reported only differences in test results between patients with and without history of HR. In addition, no report has shown that the test is useful for the prediction of drug-induced HR through longitudinal monitoring of changes in basophil activation. We evaluated the CD203c expression in 6 patients before and after the onset of HR. All patients with grade 2-4 anaphylaxis had increased CD203c+ basophils on the day before HR when their blood samples were exposed to $50 \mu \mathrm{g} / \mathrm{mL}$ carboplatin for $30 \mathrm{~min}$ and/or $3 \mathrm{~h}$. It is also noteworthy that Patients 3 and 4 had increased CD203c+ basophils after exposure to carboplatin for $3 \mathrm{~h}$ but not for $30 \mathrm{~min}$. These results suggest that the test is useful for the prediction of anaphylaxis of grade 2 or more, and that both $30 \mathrm{~min}$ and $3 \mathrm{~h}$ exposure may be necessary to predict carboplatin-related HR. The discordance of test results between $30 \mathrm{~min}$ and $3 \mathrm{~h}$ exposure to carboplatin appears to reflect the difference in the mechanisms of basophil activation, immune- and non-immune-mediated mechanisms. Carboplatinrelated hypersensitivity reactions are thought to be mediated by $\operatorname{IgE},{ }^{26,27)}$ although other studies suggested platinum salts can release histamine from basophils and mast cells without an immune-mediated mechanism (pharmacological effect of platinum). ${ }^{28,29)}$

In conclusion, the results of this study suggest that the basophil activation test based on the induction of CD203c expression seems a promising simple test for the prediction of carboplatin-related HR, particularly prediction of severe anaphylaxis.

Acknowledgements The authors thank Tomoaki Kumagai and Yoshiko Kodani (R\&D Center, BML) for their assistance in the measurement of CD203c expression on basophils. This work was supported in part by a Grant-in-Aid for Scientific Research from the Ministry of Education, Culture, Sports, Science and Technology of Japan and The Research Foundation for Pharmaceutical Sciences.

Funding Source The authors received no specific funding for this project.

\section{REFERENCES}

1) Markman M, Kennedy A, Webster K, Elson P, Peterson G, Kulp B, Belinson J. Clinical features of hypersensitivity reactions to carboplatin. J. Clin. Oncol., 17, 1141-1145 (1999).

2) Schwartz JR, Bandera C, Bradley A, Brard L, Legare R, Granai $\mathrm{CO}$, Dizon DS. Does the platinum-free interval predict the incidence or severity of hypersensitivity reactions to carboplatin? The experience from Women and Infants' Hospital. Gynecol. Oncol., 105, 81-83 (2007).

3) Navo M, Kunthur A, Badell ML, Coffer LW 2nd, Markman M, Brown J, Smith JA. Evaluation of the incidence of carboplatin hypersensitivity reactions in cancer patients. Gynecol. Oncol., 103, 608-613 (2006).

4) Sugimoto $H$, Iwamoto $T$, Murashima $Y$, Tabata $T$, Sagawa N, Okuda M. Risk factors contributing to the development of carboplatinrelated delayed hypersensitivity reactions in Japanese patients with gynecologic cancers. Cancer Chemother. Pharmacol., 67, 415-419 (2011).

5) Koshiba H, Hosokawa K, Kubo A, Miyagi Y, Oda T, Miyagi Y, Watanabe A, Honjo H. Incidence of Carboplatin-related hypersensitivity reactions in Japanese patients with gynecologic malignancies. Int. J. Gynecol. Cancer, 19, 460-465 (2009).

6) Markman M, Zanotti K, Peterson G, Kulp B, Webster K, Belinson J. Expanded experience with an intradermal skin test to predict for the presence or absence of carboplatin hypersensitivity. J. Clin. Oncol., 21, 4611-4614 (2003).

7) Leguy-Seguin V, Jolimoy G, Coudert B, Pernot C, Dalac S, Vabres $\mathrm{P}$, Collet E. Diagnostic and predictive value of skin testing in platinum salt hypersensitivity. J. Allergy Clin. Immunol., 119, 726-730 (2007).

8) Marone G, Triggiani M, de Paulis A. Mast cells and basophils: friends as well as foes in bronchial asthma? Trends Immunol., 26, 25-31 (2005).

9) Boumiza R, Debard AL, Monneret G. The basophil activation test by flow cytometry: recent developments in clinical studies, standardization and emerging perspectives. Clin. Mol. Allergy, 3, 9 (2005).

10) Ebo DG, Sainte-Laudy J, Bridts $\mathrm{CH}$, Mertens $\mathrm{CH}$, Hagendorens MM, Schuerwegh AJ, De Clerck LS, Stevens WJ. Flow-assisted allergy diagnosis: current applications and future perspectives. $\mathrm{Al}$ lergy, 61, 1028-1039 (2006).

11) Hausmann OV, Gentinetta T, Bridts CH, Ebo DG. The basophil activation test in immediate-type drug allergy. Immunol. Allergy Clin. North Am., 29, 555-566 (2009).

12) Romano A, Torres MJ, Castells M, Sanz ML, Blanca M. Diagnosis and management of drug hypersensitivity reactions. J. Allergy Clin. Immunol., 127 (Suppl.), S67-S73 (2011).

13) de Weck AL, Sanz ML, Gamboa PM, Aberer W, Bienvenu J, Blanca M, Demoly P, Ebo DG, Mayorga L, Monneret G, Sainte-Laudy J. Diagnostic tests based on human basophils: more potentials and perspectives than pitfalls. Int. Arch. Allergy Immunol., 146, 177-189 (2008).

14) Abuaf N, Rostane H, Rajoely B, Gaouar H, Autegarden JE, Leynadier F, Girot R. Comparison of two basophil activation markers CD63 and CD203c in the diagnosis of amoxicillin allergy. Clin. Exp. Allergy, 38, 921-928 (2008).

15) Sanz ML, Gamboa PM, Mayorga C. Basophil activation tests in the evaluation of immediate drug hypersensitivity. Curr. Opin. Allergy Clin. Immunol., 9, 298-304 (2009).

16) De Week AL, Sanz ML, Gamboa PM, Aberer W, Sturm G, Bilo MB, Montroni M, Blanca M, Torres MJ, Mayorga L, Campi P, Manfredi M, Drouet M, Sainte-Laudy J, Romano A, Merk H, Weber JM, Jermann TM; ENDA (European Network for Drug A1lergy). Diagnosis of immediate-type beta-lactam allergy in vitro by flow-cytometric basophil activation test and sulfidoleukotriene 
production: a multicenter study. J. Investig. Allergol. Clin. Immunol., 19, 91-109 (2009).

17) Aranda A, Mayorga C, Ariza A, Doña I, Rosado A, Blanca-Lopez $\mathrm{N}$, Andreu I, Torres MJ. In vitro evaluation of IgE-mediated hypersensitivity reactions to quinolones. Allergy, 66, 247-254 (2011).

18) Leysen J, Bridts CH, De Clerck LS, Vercauteren M, Lambert J, Weyler JJ, Stevens WJ, Ebo DG. Allergy to rocuronium: from clinical suspicion to correct diagnosis. Allergy, 66, 1014-1019 (2011).

19) Viardot-Helmer A, Ott H, Sauer I, Merk HF. Basophil activation test as in vitro assay for cisplatin allergy. Hautarzt, 59, 883-884 (2008).

20) Boumiza R, Monneret G, Forissier MF, Savoye J, Gutowski MC, Powell WS, Bienvenu J. Marked improvement of the basophil activation test by detecting CD203c instead of CD63. Clin. Exp. Allergy, 33, 259-265 (2003).

21) Nagao M, Hiraguchi $Y$, Hosoki $K$, Tokuda R, Usui T, Masuda S, Yamaguchi M, Fujisawa T. Allergen-induced basophil CD203c expression as a biomarker for rush immunotherapy in patients with Japanese cedar pollinosis. Int. Arch. Allergy Immunol., 146 (Suppl. 1), 47-53 (2008).

22) Sampson HA. Anaphylaxis and emergency treatment. Pediatrics, 111, 1601-1608 (2003).

23) Kleine-Tebbe J, Erdmann S, Knol EF, MacGlashan DW Jr, Poulsen LK, Gibbs BF. Diagnostic tests based on human basophils: potentials, pitfalls and perspectives. Int. Arch. Allergy Immunol., 141, 79-90 (2006).
24) Kvedariene V, Kamey S, Ryckwaert Y, Rongier M, Bousquet J, Demoly P, Arnoux B. Diagnosis of neuromuscular blocking agent hypersensitivity reactions using cytofluorimetric analysis of basophils. Allergy, 61, 311-315 (2006).

25) Gómez E, Blanca-Lopez N, Torres MJ, Requena G, Rondon C, Canto G, Blanca M, Mayorga C. Immunoglobulin E-mediated immediate allergic reactions to dipyrone: value of basophil activation test in the identification of patients. Clin. Exp. Allergy, 39, 1217-1224 (2009).

26) Weidmann B, Mülleneisen N, Bojko P, Niederle N. Hypersensitivity reactions to carboplatin. Report of two patients, review of the literature, and discussion of diagnostic procedures and management. Cancer, 73, 2218-2222 (1994).

27) Cleare MJ, Hughes EG, Jacoby B, Pepys J. Immediate (type I) allergic responses to platinum compounds. Clin. Allergy, 6, 183-195 (1976).

28) Kook H, Kim KM, Choi SH, Choi BS, Kim HJ, Chung SY, Choi SJ, Hwang TJ. Life-threatening carboplatin hypersensitivity during conditioning for autologous PBSC transplantation: successful rechallenge after desensitization. Bone Marrow Transplant., 21, 727-729 (1998).

29) Moreno-Ancillo A, Domínguez-Noche C, Gil-Adrados AC, Cosmes PM, Duarte I, Mateos Y. Anaphylactoid reaction to carboplatin: successful "desensitization." Allergol. Immunopathol. (Madrid), 31, 342-344 (2003). 